\title{
Remote Management of left ventricular device assisted patients
}

\author{
Giorgos Aristomenopoulos \\ VELTI S.A \\ Athens, Greece
}

\author{
Rossella Fontana, Monica Vatteroni, \\ Giuseppe Tortora \\ The BioRobotics Institute, \\ Scuola Superiore Sant'Anna, \\ Pisa, Italy
}

\begin{abstract}
Ventricular Assist Devices (VADs) have been lately considered as an efficient destination therapy for heart disease, achieving remarkable survival scores. This paper presents an integrated end-to-end VAD patient architecture consisting of a web-based HL7 compatible Specialist Monitoring Application, an Android-based Patient Monitoring Application and a portable embedded Auto-Regulation Unit is described, delivering for the first time an interconnected solution that not only combines the characteristics of EHR systems and allows the efficient monitoring of patients' and VAD status, but also enables the remote control, configuration and auto-regulation of any VAD.
\end{abstract}

Keywords: vad; remote patient management; aito-regulation.

\section{INTRODUCTION}

According to the European Heart Network and European Society of Cardiology, diseases of the heart and circulatory system (cardiovascular disease or CVD) are the main cause of death in Europe, accounting for over 4 million deaths each year [1]. Although at present heart transplantation is still regarded as the gold standard in the surgical treatment of terminal heart disease, Ventricular Assist Devices (VAD) - electromechanical circulatory devices that are used to partially or completely replace the function of a failing heart - have attracted significant attention over the last years due to increased survival rates of implanted patients. The 6-month survival in bridge-to-transplant patients rose to $93 \%$ in 2011 , whereas in 2008 a rate of $82 \%$ had been reported [2]. Moreover the continuous advances in biomedicine, materials and fabrication favor the use of VADs even as destination therapy, ensuring the long term survival of the patient [3]. A beyond state of the art example of the modern capabilities of the VAD is presented by CircuLite $\mathrm{GmbH}$ (Aachen, Germany) and em-tec $\mathrm{GmbH}$ (Finning, Germany), featuring integrated flow and pressure sensors, transcutaneous energy transfer (TET) and telemetry (TEL) modules [4]. This does not only imply the abolition of cables, causing life-threatening inflammation and sepsis sideeffects, but also facilitates the design and development of novel wireless monitoring and management systems, ranging from extracorporeal sophisticated control units residing by the patient, to complex multimodal systems residing at the doctors' premises. In particular, an autoregulation unit (ARU) has been designed and proposed, offering a dedicated portable autonomous controller capable of dynamically and wirelessly regulating the operational and functional parameters of the VAD. However, despite the promising results of such a system in terms of efficiently controlling the sensorized VAD [5], or integrating with a multi-functional hybrid simulator towards emulating a wide range of pathologies for testing VAD performance [6], the critical eye and the experience of a highly trained specialist that is capable of dynamically and remotely intervening on the VAD configuration is missing.

This paper describes an integrated end to end VAD implanted patient treatment architecture enabling a) specialists to remotely monitor and manage the operation of the pump, $b$ ) specialists to remotely administer patients, c) patients to efficiently monitor their progress and status at home, and d) VADs to adapt and optimize their operation on the fly. Specifically Sections II to IV provide an overview of the Patient Monitoring Application, the Specialists' Monitoring Application and the Auto-Regulation Unit respectively. Section $\mathrm{V}$ illustrates the overall integrated architecture, while Section VI sketches an indicative scenario of the platform's usage and features. Finally Section VII concludes this document.

\section{PATIENT MONITORING APPLiCATION}

Patient Monitoring Application (PMA) is an Android 4.x application that eases and simplifies numerous daily activities of VAD patients and specialists by a) enabling the proficient monitoring of patients' activities, b) enabling specialists and care givers to remotely monitor patients' progress, c) providing early detection warning systems and d) reducing the unnecessary hospital visits. In the following a brief overview of the PMA functionalities is described. The main page that the patient encounters when launching the PMA essentially provides access to the complete list of offered features, while also providing a quick status overview of patient's pending issues. Specifically PMA includes:

- Medication allows patients to view their daily and monthly drug prescription plan, as well as to mark medication as taken, eliminating drug abuse while also informing the specialist of patient's compliance with the treatment plan.

- Appointments offer a simple reminder service and tracking mechanism for scheduled hospital visits. Appointments are set by specialists and are automatically update in patients' devices.

- Chat provides an instant messaging exchange service, allowing the imminent of the patient and the care giver.

- Measurement Plan allows patients to view their daily measurement plan as set by the specialist. 


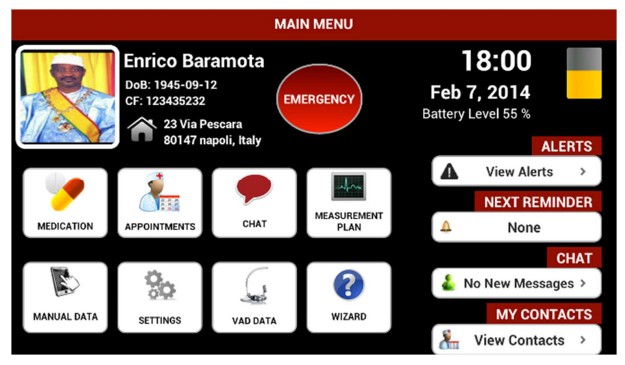

Figure 1 - Patient Monitoring Application Interface

- Manual Data allows patients to insert the results of their daily measurements. History records are also kept for future reference, while all measurements are automatically forwarded to the specialist. Measurement can be recorded either by manually inputting them, or using a Continua Health Alliance (CHA) certified device. PMA supports: Weight, Blood Pressure, Temperature, Quality of Life, Pulse Oximetry, Fluid intake/Diuresis, VAD data and INR.

- Settings allow the configuration of various settings of the PMA, like Bluetooth connections, notifications and reminders, as well as Localization settings.

- VAD Data displays the latest measurements acquired from the ARU, providing a brief overview of the VAD status.

- Help Wizard provides an interactive tutorial that assists patients in familiarizing with PMA's functionalities. The program guides the patient throughout the whole process, highlighting the proper buttons.

- Alerts display the latest clinical alerts as triggered by the patient's out of bound vital signals, e.g. fever, tachycardia etc.

- Reminders provide a list of upcoming reminders with regards to the patient's prescribed measurement and drug plan.

- Chat notifier presents any unread or missed conversations.

- My contacts display contact information, useful in cases of emergency or consultation on clinical matters.

- Emergency Button provides quick information about the patient and his treating specialist. Useful in emergency situations when the First Aid team arrives.

\section{SPECIALIST MONITORING APPLICATION}

The Specialist Monitoring Application (SMA) is an HL7 compliant web platform that provides doctors, clinicians, nurses and researchers enhanced features and increased flexibility, facilitating the complete monitoring and treatment procedure of VAD patients. It presents and meaningfully combines data from multiple patient monitoring systems (as well as other measurements submitted by the specialist, e.g. lab measurements), on a 24-hour base. More importantly, the specialist and the medical team responsible for a patient are informed in real time for alarming or threatening events related to the patient's health or the VAD's, while via a completely personalized (per specialist and per patient) threshold-based mechanism for alerts generation, the early recognition of complications is permitted. The specialist is able to exchange instant messages with his patients and last but not least the specialist is capable of remotely controlling the operation of

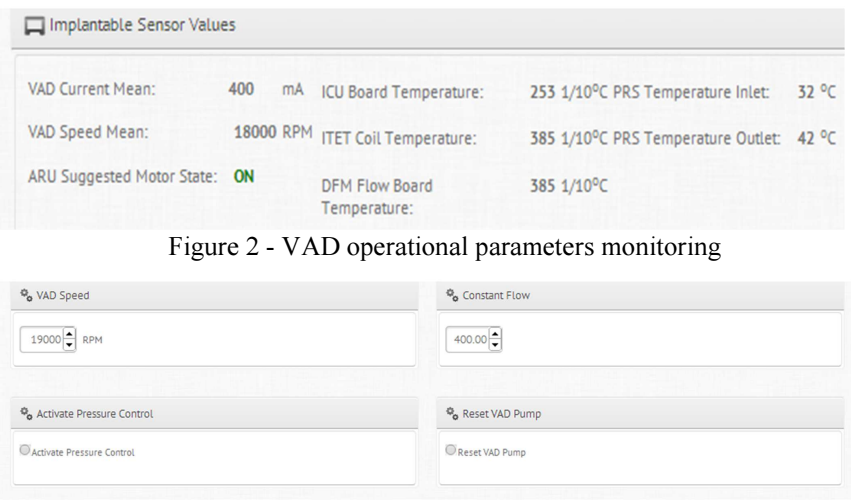

Figure 3 - VAD remote control

the $V A D$. Specifically the $\boldsymbol{S M A}$ main page a specialist provides imminent access to all platform features including:

- The Patient list that contains the all patients, the specialist has access to (anonymized or not). Selecting one will redirect to the Patient Record Main page as described in the following.

- The list of Alerts, describing any potential alarm or critical condition of patients' vital signals, as triggered either by the patients' monitoring application (e.g. high temperature) or by the implantable pump (e.g. low blood flow).

- The Instant messaging module, enabling the specialist to directly interface and communicate with patients.

- The Decision Support System, assisting the specialists into taking the most appropriate treatment decision, as derived by sophisticated statistical analysis of similar cases.

Moreover, The Patient Record Main of a specific patient consists of a number of tabs which present different information regarding the selected patient:

- Patient Visit information, providing details, measurements and checkup results, of Preoperative Visits, Intervention In and Out Visits, Hospital Discharge, Early Postoperative Visits (1-7 days) and Long Term Postoperative Visits (1-24months)

- Manual Measurement Data, containing patients' prescription plan of manual or sensor measurements. Such measurements include for example weight, measured manually by the patient, or via Bluetooth using a CHA certified device.

- Patient Progress, containing the summary of patient's monitored data, providing values and graphs from data originating from a) the wearable platform, b) the implantable platform (Figure 2), or c) CHA connected devices

Device Administration, offering the specialist the possibility to remotely configure the PMA features that will be active (e.g. chat, alerts, Manual measurements etc.), send commands to VAD (e.g. alter pump speed, flow etc) (Figure 3), or even set VAD alarming thresholds (e.g. max flow pressure).

- Clinical Knowledge Editor, enabling specialists to create and modify clinical guidelines for use in the alerting mechanism of the PMA.

- Drugs, enabling the specialist to create and modify patient's prescription plan. Moreover, the proper monitoring of 
patient's drug intakes is also provided, assuring the proper execution of the prescribed medication plan.

- Lab Exams, providing a robust parser that is capable of parsing PDF files containing lab results, automating the data input procedure.

\section{AutO REGULATION UNIT}

The Autoregulation Unit (ARU) is an embedded portable device carried by the patient, responsible for wirelessly controlling and monitoring the performance of the implanted sensorized VAD. In order to fit the autoregulation needs, the hardware design of the ARU has been developed by integrating standard interfaces (such as serial and standard Bluetooth ports) and implementing general rules and control actions which are performed and managed by a dedicated FPGA (Field Programmable Gate Array). The ARU hardware integrates the FPGA development board and a custom printed circuit board in a single box of $170 \mathrm{~mm} \times 135 \mathrm{~mm} \times 45 \mathrm{~mm}$, which can be worn easily by the patient. The use of the FPGA increases the system flexibility and efficiency, allowing the interconnection with any VAD either by complying to the ARU API, or by appropriately modifying the firmware in terms of control tasks and algorithms to comply with vendors requirements.

The firmware implemented on the FPGA handles all the functionalities of memory management, Bluetooth interface communication towards the PMA, serial communication to the sensorized VAD, UART/BL interface to wearable portable devices for the acquisition of physiological parameters. The VHDL (VHSIC Hardware Description Language, where VHSIC is the acronym of Very High Speed Integrated Circuits) is the hardware language used to implement the firmware which makes the proposed system universal.

The ARU also interfaces a local control device for visualizing VAD data locally. The unit, called UCI (User Control Interface) is composed by an LCD display which shows real-time data from the sensorized VAD and pushbuttons which allow the VAD speed setting and resetting.

The main feature of the ARU consists on the possibility of implementing algorithms for adapting real-time the VAD operation to the physiological needs and activity of the patient. The implemented algorithms are based on traditional control rules and dynamic sensor data which are monitored, stored, processed and transferred to the PMA/SMA. In particular the following two control algorithms have been realized:

- Proportional-integrative (PI) control of the pump speed in order to achieve the desired flow value;

- Pressure variations control with alert generation in case of fast and /or repetitive changes in the pressure value measured in input of the pump.

Moreover exploiting the built-in control algorithms, the ARU as its name implies is capable of auto regulating the VAD operation sensing and reacting to various triggers. Specifically:

- Auto-regulation to the desired flow level: When the flow control is activated, the algorithm compares the current flow value coming from the pump with the desired value (previously set by the specialist). When a difference is present,
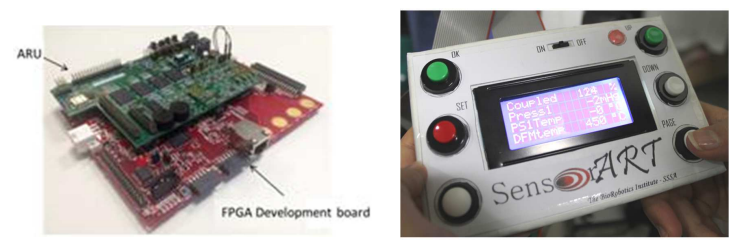

Figure 4 - The ARU (left) and the UCI (right)

the PI controller changes the speed towards reaching the desired flow.

- Auto-regulation suction avoidance: A suction event is due to the collapse of the inflow tube caused by an excessive decrease of the inflow pressure with respect to the atmospheric pressure. This compromises the correct functioning of the implanted pump. Thus via detecting suction events (based on the control of the inlet pressure beyond a threshold) the ARU resets the pump, recovering to the normal working condition.

Auto-regulation performance of the ARU has been successfully demonstrated using both an on bench simulator [5] and an advanced hybrid numerical simulator [6].

Moreover, safety is also guaranteed by the built-in ARU alarms. In case some value exceeds the thresholds set by the specialist via SMA, an alarm will be triggered and forwarded to the treating specialist for imminent action.

\section{INTEGRATED ARCHITECTURE}

Figure 5 presents an overview of the architecture integrating all involved modules enabling their direct communication. Specifically:

- SMA interfaces with external HIS (Hospital Information Systems) complying to the HL7 v2.0 messaging exchange protocol. Mirth Connect acts as a proxy collecting HL7 messages (e.g. patient profiles, demographic data), translating them and pushing the information to the SMA database.

- SMA interfaces with PMA over the internet via a set of RESTful web services enabling them to synchronize the latest delay-tolerant (e.g. measurement plans) and real-time (e.g. Alerts) patient data, update and apply clinical rules to patients, remotely configure PMA settings and push operational thresholds and commands to be forwarded to VAD.

- PMA interfaces with $A R U$ via Bluetooth Serial Port Profile (SPP). PMA acts as a bridge collecting monitoring information from ARU and commands and alerts from SMA and forwards them to SMA and ARU respectively. Thus, upon successful pairing ARU sends to PMA packets of 906 Bytes containing more than 30 monitored VAD parameters, updated with a frequency of $60 \mathrm{~Hz}$. In a similar fashion ARU forwards to PMA 77-Byte packets containing wearable data updated with a frequency of $50-200 \mathrm{~Hz}$. Moreover a proprietary asynchronous API enables PMA to send VAD operational commands and thresholds as instructed by SMA, while to collect VAD alarms as generated by ARU and forward them to SMA.

- PMA interfaces with Medical Sensors certified by CHA via exploiting the IEEE 11703, the only certified standard describing the communication between medical and health care devices and with external computer systems. This ensures not 




Figure 1 - Integrated Architecture

only that currently available compliant medical devices are supported, but also that any future ones, independently of the vendor will also be. To our knowledge the developed API is the first complete Android-based implementation supporting out-ofthe box a multiplicity of off-the-shelf CHA devices.

- ARU interfaces with wearable devices towards monitoring physiological parameters via a dedicated Bluetooth module. Depending on the attached device, the protocol, written in VHDL language, is appropriately adapted. This open and flexible approach favors the interconnection with any commercial device without need of any hardware changes.

- ARU interfaces with VAD wirelessly based on a 3.3V logic. The complete set of monitored physical parameters is transferred from the sensorized VAD to the ARU via Bluetooth. Data are acquired synchronously from the VAD that generates packets at $60 \mathrm{~Hz}$ rate. In case VAD operational commands are present originated from SMA or UCI - they are forwarded to the VAD at the end of each data packet altering the VAD operation on the fly.

It is important to highlight the flexibility and interoperability of the SMA-PMA-ARU architecture and relevant APIs. HL7 interoperability enables the interconnection with any $3^{\text {rd }}$ party hospital system, while most importantly we define and provide a concrete API enabling the efficient management and monitoring of any sensorized VAD. For the first time, the foundations of a device-independent, holistic, end-to-end patient management framework are set, providing the proposed architecture unique competitive advantages and eliminating the burden of software development from VAD manufactures. In the following the efficacy of the developed integrated architecture is illustrated via the presentation of an imaginary, though likely scenario depicting the life of an implanted VAD patient.

\section{A DAY IN MR. BARAMOTA'S LIFE}

Mr. Baramota has just been implanted a sensorized pump. After the surgery, the specialist creates the patient profile in the SMA and remotely sets the initial VAD parameters via the SMA environment based on the suggestions of the decision support tools [7]. The patient is provided with a tablet (PMA), a sensorized t-shirt, CHA compatible devices, a belt equipped with the ARU and the extracorporeal part of the implantable platform (TET and TEL system).

Once at home Mr. Baramota in line with the physician's instructions will wear the wearable and the belt equipped with the ARU. This way his physiological (ECG, respiratory and movement activity) and VAD operational parameters (flow, pressure, speed, current, etc), will be continuously monitored and the recorded. All the data are sent and stored to the ARU, while as soon as PMA is connected to the ARU, data are automatically transferred to the integrated repository. Moreover, using PMA and external CHA-compatible devices, he can also wirelessly upload additional information like his temperature, water assumption, weight, blood pressure etc.

At that point, the specialist can remotely monitor the patient's status and progress via SMA, checking all collected implantable and wearable information. In case monitoring values exceed the thresholds, alarms will be triggered prompting them for action. The patient can follow the advices that automatically appear at PMA, while the specialist can even dynamically and remotely change VAD parameters through SMA towards mitigating the alarm cause. The latter is achieved via the cooperation of the appropriate communication modules, and specifically SMA communicates the commands to PMA which in turn sends them to the ARU via Bluetooth. The ARU communicates with the implantable platform via the telemetry system and the VAD parameters are modified accordingly.

Moreover, in case of high-level alarms, using smart autoregulation algorithms, the ARU dynamically adjusts the VAD operational characteristics, responding to patient's needs mitigating adverse events and reducing hospitalization, providing on-the-fly treatment capabilities.

\section{CONCLUSIONS}

A novel integrated end-to-end VAD patient management system has been presented covering the complete treatment and monitoring procedures of VAD implanted patients. The modular and flexible architecture of the proposed solution favors the integration of the system with any HL7-compatible HIS system and any VAD requesting minimal modifications.

The efficacy and robustness of the proposed architecture has been successfully tested and evaluated during in vitro (Finning, Germany) and in vivo (Pisa, Italy) tests verifying its applicability and accuracy, yet most importantly paving the way of a remotely controlled cable-free VAD system.

\section{REFERENCES}

[1] European Cardiovascular Disease Statistics 2012, EuroHeart II, European Heart Network and European Society of Cardiology, September 2012

[2] F. Pagani, LW Miller, SD Russell, et al. "Extended Mechanical Circulatory Support With a Continuous-Flow Rotary Left Ventricular Assist Device", J Am Coll Cardiol. vol 54, iss 4, Elsevier, 2009.

[3] T. Hrobowski and D. E. Lanfear, "Ventricular assist devices: is destination therapy a viable alternative in the non-transplant candidate?", Current Heart Failure Reports, vol. 10, iss 1, Springer, 2013

[4] A. Verbeni, R. Fontana, M. Silvestri, et al., "An innovative adaptive control strategy for sensorized Left Ventricular Assist Devices", IEEE Trans. on Biomedical Circuits and Systems, Sept. 2014.

[5] R. Fontana, M. Silvestri, G. Tortora, et al., "An Autoregulation Unit for enabling adaptive control of sensorized left ventricular assist device", in Proc. IEEE 35th Annu. Int. Conf. Eng. Med. Biol. Soc (EMBC), 2014

[6] G. Tortora, R. Fontana, L. Fresiello, et al., "Experimental integration of Autoregulation Unit for left ventricular assist devices in a cardiovascular hybrid simulator", In Proc IEEE 35th Annu. Int. Conf. Eng. Med. Biol. Soc (EMBC), 2014

[7] EC. Karvounis, MG Tsipouras, AT Tzallas, et al., "A decision support system for the treatment of patients with ventricular assist device support", Methods Inf Med. 2014;53(2):121-36. 